\title{
Intrathecal baclofen treatment in dystonic cerebral palsy: a randomized clinical trial: the IDYS trial
}

Laura A Bonouvrié ${ }^{*}$, Jules G Becher ${ }^{1}$, Johannes SH Vles ${ }^{2}$, Karin Boeschoten ${ }^{1}$, Dan Soudant ${ }^{2}$, Vincent de Groot ${ }^{1}$, Willem JR van Ouwerkerk ${ }^{3}$, Rob LM Strijers ${ }^{4}$, Elisabeth Foncke ${ }^{5}$, Joke Geytenbeek ${ }^{1}$, Peter M van de Ven ${ }^{6}$, Onno Teernstra ${ }^{7}$ and $R$ Jeroen Vermeulen ${ }^{8}$

\begin{abstract}
Background: Dystonic cerebral palsy is primarily caused by damage to the basal ganglia and central cortex. The daily care of these patients can be difficult due to dystonic movements. Intrathecal baclofen treatment is a potential treatment option for dystonia and has become common practice. Despite this widespread adoption, high quality evidence on the effects of intrathecal baclofen treatment on daily activities is lacking and prospective data are needed to judge the usefulness and indications for dystonic cerebral palsy. The primary aim of this study is to provide level one clinical evidence for the effects of intrathecal baclofen treatment on the level of activities and participation in dystonic cerebral palsy patients. Furthermore, we hope to identify clinical characteristics that will predict a beneficial effect of intrathecal baclofen in an individual patient.

Methods/Design: A double blind placebo-controlled multi-center randomized clinical trial will be performed in 30 children with dystonic cerebral palsy. Patients aged between 4 and 25 years old with a confirmed diagnosis of dystonic cerebral palsy, Gross Motor Functioning Classification System level IV or V, with lesions in the cerebral white matter, basal ganglia or central cortex and who are eligible for intrathecal baclofen treatment will be included. Group A will receive three months of continuous intrathecal baclofen treatment and group B will receive three months of placebo treatment, both via an implanted pump. After this three month period, all patients will receive intrathecal baclofen treatment, with a follow-up after nine months. The primary outcome measurement will be the effect on activities of and participation in daily life measured by Goal Attainment Scaling. Secondary outcome measurements on the level of body functions include dystonia, spasticity, pain, comfort and sleep-related breathing disorders. Side effects will be monitored and we will study whether patient characteristics influence outcome.
\end{abstract}

Discussion: The results of this study will provide data for evidence-based use of intrathecal baclofen in dystonic cerebral palsy.

Trial registration: Nederlands Trial Register, NTR3642

Keywords: Cerebral palsy, Dystonia, Dyskinesia, Goal attainment scaling, Intrathecal baclofen, Randomized controlled trial

\footnotetext{
* Correspondence: I.bonouvrie@vumc.nl

'Department of Rehabilitation Medicine, VU University Medical Center,

Postbus 7057, 1007, MB Amsterdam, The Netherlands

Full list of author information is available at the end of the article
} 


\section{Background}

\section{Dystonic cerebral palsy}

Cerebral palsy (CP) is a group of disorders caused by non-progressive disturbances that occurred in the developing fetal or early infant brain. The classification of cerebral palsy includes the classical neurological terms for central motor disorders: spasticity, dyskinesia and ataxia [1,2]. Dyskinesia can be further differentiated into dystonia and choreoathetosis [2]. Dystonia is described as an abnormal pattern of posture and/or movement that is involuntary, uncontrolled, recurring and occasionally stereotyped. These movements can interfere with daily care and may be painful and uncomfortable. The dyskinetic form of cerebral palsy, including the dystonic form, is in most patients caused by lesions in the basal ganglia. Additional lesions of the central cortex are found in some cases. This type of brain damage is a common pattern in asphyxiated infants born at term [3].

\section{Treatment}

The results of pharmacological treatment of severe dystonic $\mathrm{CP}$ have been rather disappointing. Positive effects on dystonia with levodopa, anticholinergic drugs or muscle relaxants including benzodiazepines and baclofen, have been reported by some authors [4,5]. In addition, Albright and co-workers described an antidystonic effect of intrathecal baclofen (ITB) treatment. Despite the fact that studies on the effects of ITB on dystonic cerebral palsy are limited in number and the level of evidence is low, ITB is now common practice in the treatment of severe dystonic CP.

Not all patients are eligible for ITB treatment. The following criteria apply: 1 . The etiology is preferably known; 2. Management of aggravating factors, such as pain and discomfort, should be optimal; 3. Other treatment options should have been explored. Oral pharmacological treatment with levodopa, anticholinergic drugs or muscle relaxants including benzodiazepines and baclofen must have been attempted and must have resulted in high oral dosages with either unacceptable side effects or insufficient efficacy; 4. The movement disorder should be so severe that it interferes with activities of daily life or quality of life; 5 . Treatment goals should be clear and applicable and, to avoid disappointment, it is important that patients and parents understand these goals; 6. Patients and parents should be motivated and able to adhere to the requirements of treatment, such as the frequent pump fillings and checkups in the outpatient clinic; 7. Patients should have sufficient body size to allow pump implantation [6].

\section{Objectives}

The primary objective of the present study is to show whether ITB treatment improves activities of and participation in daily life (for example dressing, transfer, sitting in a wheelchair, hygienic care, speech) in dystonic $\mathrm{CP}$ patients. Certain patient characteristics, such as the location and severity of MRI lesions and Gross Motor Functioning Classification Score (GMFCS) level, might influence the effects of ITB treatment in patients with dystonic CP. Therefore, we will study whether individual patient characteristics (GMFCS, gender, age, MRI findings and co-medication) influence outcome and could be used in determining the indication for ITB in future patients. A secondary objective is to provide evidence for the effect of ITB on the level of body functions. The relevant clinical questions to be addressed are: 1 . Does ITB decrease dystonia? 2. Does ITB decrease spasticity in dystonic patients? 3. Does ITB decrease pain? 4. Does ITB increase comfort? 5. Does ITB influence screening results of sleep-related breathing disorders? 6 . What are the side effects of ITB?

\section{Methods/Design \\ Study design}

The design of the study is a double blind placebocontrolled multi-center randomized clinical trial. It will be conducted in the VU University Medical Center (VUMC) in Amsterdam and the Maastricht University Medical Center (MUMC) in Maastricht (both in the Netherlands). The Medical Ethical Committee of the VU University Medical Center approved the study. In both centers local practicability was granted subsequently. Subjects will be included over a period of two and a half years and they will participate in the study for one year. Figure 1 shows the flow scheme for subjects and timing of measurements throughout the study.

\section{Participants}

Thirty subjects will be recruited from the outpatient clinics of the pediatric neurology and pediatric rehabilitation departments of the VUMC and the MUMC. Table 1 shows the inclusion and exclusion criteria. We selected ages between 4 and 25 years old because older patients often show secondary complications, such as contractures, that could introduce greater variation into both the effects of treatment and treatment goals. Furthermore, to achieve a homogeneous patient group, only GMFCS IV and V (non-walkers) will be included. All patients and/or their caregivers will sign an informed consent form before participating in the study.

\section{Sample size calculation}

We will use goal attainment scaling (GAS) as our primary outcome measure. The within group change for the placebo group is anticipated to be 0 , with a standard deviation (SD) of 8.5. For the intrathecal baclofen group, the within group change is anticipated to be 12.5 , with a 


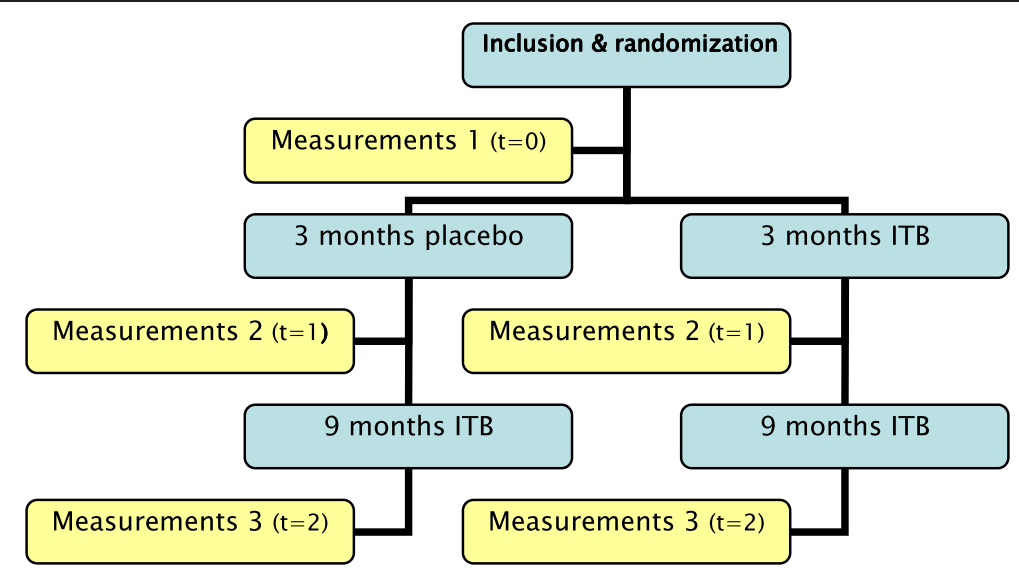

Figure 1 Flow chart subjects.

SD of 10 . To achieve a power of $90 \%$ when testing for a difference in means using a two-tailed independent samples t-test and a significance level of 5\%, a total of 13 subjects per group will be needed.

In a previous study in the VU University Medical Center, 4 infections occurred in 34 pump implantations [7]. Ward and coworkers reported pump removal in 5.9\% of the cases with infections following implantation [8]. Extrapolating these numbers, inclusion of 30 patients is expected to lead to 3.5 infections and 1.2 pump removals. To prevent the study from becoming underpowered, due to unexpected protocol violations or subject dropout due to complications other than those related to intrathecal baclofen treatment, we will include 15 subjects per group.

\section{Intervention}

Included subjects will be randomized in two groups. Group 1 will receive placebo treatment via an implanted micro-infusion pump for three months. Group 2 will receive ITB treatment via an implanted micro-infusion

Table 1 Summary of inclusion and exclusion criteria

\begin{tabular}{ll}
\hline Inclusion criteria & Exclusion criteria \\
\hline - Dystonic cerebral palsy & $\begin{array}{l}\text { C Contra-indications for general } \\
\text { anesthesia }\end{array}$ \\
- GMFCS IV or V & - Contra-indications for baclofen \\
- Eligible for ITB treatment using & - Oral pharmacological treatment \\
criteria of common practice & is sufficient \\
- Lesions on MRI (cerebral white & - Inadequate knowledge of \\
matter, basal ganglia, central cortex) & Dutch language \\
- Aged 4 to 25 years old & - Deep brain stimulation \\
- Able and willing to complete & - Ventriculoperitoneal drain \\
study protocol & \\
- Consensus about inclusion & - Other disorders interfering with \\
& treatment \\
\hline
\end{tabular}

pump for three months. In our opinion, it is unethical to continue placebo treatment for more than three months. If interim analysis shows that subjects in one of the groups have significant disadvantages compared to the other group, the study will be prematurely terminated. On the other hand, we know from experience that dosages for some patients are still being modified three months after starting treatment. As a consequence, the effect of treatment in these patients may not yet be optimal, which may then result in no detectable effect of ITB treatment at that point. We will try to reduce this possible effect through frequent dose modification. Patients will be seen once or twice weekly to increase their dosage, until either a stable dosage or a maximal dosage of $800 \mu \mathrm{g} /$ day is achieved.

Subjects will be assessed at three months and the effect of treatment in the two groups compared. Since determining the most beneficial pump setting can take more than three months and because the initial effect may recede in some cases, all subjects will receive subsequent ITB treatment for nine months. Following this 9 month period, clinical evaluation will be performed in order to determine long-term effects (see Figure 1. Flow Chart of Subjects).

\section{Dosage}

Patients with dystonia seem to require larger ITB doses than the doses used to treat spasticity [9]. Albright and colleagues noticed a change in dystonia only after 4 days of continuous infusion [10]. The effective dose of ITB was in the range of 350 to $750 \mu \mathrm{g} /$ day [9-11]. Since a screening period will not be included, we do not know how subjects will respond and which dose will be sufficiently effective. Therefore, the starting dosage will be $50 \mu \mathrm{g} / 24 \mathrm{~h}$.

Dosage can be increased by $10-20 \%$ daily. When subjects are discharged from the hospital following pump 
implantation, they will be seen once a week or once every two weeks for dosage modification. After three weeks of increasing dosage, with disappearance of the effect of the increase, daily boluses will be administered to a maximum of four times a day, with a minimal interval of four hours. For the blinded part of the study, a maximum dosage of $800 \mu \mathrm{g} /$ day will be administered. This is a reasonable estimation of the maximal dosage after three months. Since treatment during the study is blinded, the physician in charge of regular follow-up after pump implantation, including pump filling and dosage adjustments, will be unaware of the subject's allocated group. To provide optimal treatment, pump settings will be individually altered to the extent that the physician would consider necessary, based on the findings of physical examination and parental and patient interview, as were the patient not participating in the study. As a consequence, the pump settings of subjects in the placebo group will be altered as if the patient was receiving ITB treatment.

Lack of response to ITB can be caused by pump or catheter dysfunction. Placebo treatment may also cause lack of effect and for this reason no action will be taken during the first three months of the study in cases where pump or catheter dysfunction would normally be suspected. An exception will be made when the condition of the patient requires otherwise, such as in the case of signs of withdrawal. One week after pump implantation, all patients will undergo X-ray of the spine to determine the position of the catheter tip. The catheter should be placed approximately at the fourth cervical level, and at least above the level of Th1.

\section{Outcome measures}

Outcome measurements are defined on the levels of the International Classification of Functioning, Disability and Health (ICF) model of the World Health Organization (WHO). We distinguish the level of activities and participation and the level of body functions. Body functions are the physiological and psychological functions of body systems. Activities are defined as the execution of a task or action by an individual. Participation is involvement in a life situation [12].

\section{Primary outcome measure}

The primary outcome measurement will be on the level of activities and participation. Goal attainment scaling (GAS) will be used to measure the effect of ITB treatment. Using GAS, achievement of individual set goals can be quantified. This method was introduced for assessing outcomes in mental health settings and has been used in many other areas $[13,14]$. Each subject has their own outcome measure, but statistical analysis is possible because they are scored in a standardized way.
This procedure is time-consuming and requires about 45 minutes per child $[15,16]$. However, after the scale is constructed, it should be possible to score a patient within 10 minutes [17].

The procedure includes the following aspects:

1) Identification of goals:

Three main problems of daily care and function will be determined by caregivers. Goals will be set for these problems with the help of the team to ensure that goals are achievable. Goals should be specific, measurable, attainable, realistic and timely (SMART) $[13,14]$. The target activity, specific support and time period should be specified and performance should be quantified using distance, frequency or time taken to accomplish a task [14].

2) Weighing of goals:

Some goals will be more important for subjects than others [13]. Goals can be assigned a weighing score by caregivers and a difficulty score by the team [14]. We chose not to assign weighing scores since the weighted and unweighted scores are closely correlated [18]. A value of 1 is applied to 'weight' in the formula described below.

3) Definition of expected outcomes:

Several approaches are described in literature, varying from 5 to 7 point scales [14,15]. We will use a 6 point scale ranging from -3 to +2 , since we wish to include both the possibility of partially achieving the set goal and to avoid a bottoming effect. Baseline scores will be allocated as -2 . If the subject achieves the expected level, this is scored as 0 . If a subject does not achieve the expected level but shows improvement, this is scored as -1 . If they achieve more than the expected outcome, this is scored as +1 for somewhat more and +2 for much more. We chose to add a score of -3 in case of deterioration. Each goal level will be defined by the investigator so as to be as objective and observable as possible [13].

4) Scoring goal attainment:

For each assessment, one assessor will make a standardized video recording during trials for each of the three functional ability goals. The recording procedure will be identical for all measurements. Another assessor, blinded for group allocation, will rate the subject's performance from the video recordings. Although the GAS only simulates the subject's own functional setting, parents were convinced that the outcome of scaling was representative of their own setting [19].

Goal attainment scores will be recorded at baseline, after three months of blinded treatment and after nine months of ITB treatment. Goal attainment change 
scores will be determined by subtracting the baseline score from the outcome score. Subjects who achieve a GAS T-score $>50$ achieved their goals [8]. Clinical relevance will be defined as an improvement of at least two points in at least one of the three goals [19].

A single aggregated score (T-score) can be produced by a standardized mathematical formula: Overall GAS = $50+10 \sum\left(w_{i} x_{i}\right) / \sqrt{ }\left((1-\rho) \sum w_{i}^{2}+\rho\left(\sum w_{i}\right)^{2}\right) ; W_{i}$ is the weight assigned to the goal, $x_{i}$ is the numerical value achieved (between -3 and +2 ), $\rho$ (rho) is the expected correlation of the goal scales, which is normally $0.3[13,14]$.

\section{Secondary outcome measures Dystonia}

Videos of all patients will be made using the video protocol described by Monbaliu et al. [20]. Blinded therapists or physicians will assess all videotapes and rate dystonia using the Barry-Albright dystonia (BAD) scale and the Dyskinesia Impairment Scale (DIS).

The BAD scale is a five point ordinal severity scale to assess secondary dystonia in eight body regions (eyes, mouth, neck, trunk, each upper and lower extremity) [21]. Raters score dystonia as none, slight, mild, moderate or severe. A reduction of $25 \%$ or more, in comparison with the baseline score, is considered clinically significant. In our experience, interrater variability is high, but BAD scores are generally accepted as a measurement of dystonia and are widely used to assess dystonia.

Recently, a new instrument to measure dystonia in dyskinetic CP became available, the DIS [20]. It consists of two subscales: dystonia and choreoathetosis. Scoring is carried out in 12 body regions all in two conditions (rest and activity). Both duration and amplitude are evaluated. Since this is a new instrument, we will use the DIS in addition to the BAD.

\section{Electromyography}

The DIS has no external validation. We decided that Surface Electromyography (EMG) might provide an impression of muscle activity level underlying dystonia. Therefore, surface EMG measurements will be carried out to determine mean EMG activity in individually determined muscle groups and in multiple conditions such as rest and during activities.

\section{Spasticity}

The soleus Hoffmann-reflex (H-reflex) represents excitability of the neural components of the stretch reflex arc [22]. The H/M-ratio of the H-reflex represents an increased excitability of soleus motor neurons. The $\mathrm{H} /$ M-ratio is increased in subjects with spasticity due to various origins, [23] and it has been shown that the $\mathrm{H}_{\max }$ decreases significantly after ITB administration in children with spastic cerebral palsy when compared with baseline measurements. This represents a decrease in motor neuron excitability. Furthermore, the response appears to be dose-dependent [22]. Although not all children tolerate the measurements, feasibility of the H-reflex was $93 \%$ in a study by Hoving and co-workers [22].

For clinical assessment of spasticity in children with central motor disorders, the spasticity test (SPAT) is used during standard physical examination. When using the SPAT, we will follow a standardized protocol as described in the guideline for standard physical examination of children with central motor disorders [24,25]. The difference between the range of motion and the angle of catch will be used as the outcome measure for spasticity [26]. The test takes approximately 5 to $8 \mathrm{mi}-$ nutes per limb to perform [25]. This test might be difficult to perform in children and adolescents with severe dystonia and its usefulness will become evident during the study.

\section{Pain and comfort}

Parents will score pain and comfort on a visual analogue scale (VAS). The VAS is a straight $10 \mathrm{~cm}$ long horizontal block consisting of 10 smaller blocks with anchor points at 0 and 10. For pain, a score of 0 represents 'no pain' and a score of 10 represents 'the worst possible pain'. For comfort, a score of 0 will represent being 'very uncomfortable' and a score of 10 is having 'no problems at all'. If patients are able to indicate their mood, it will be scored by pointing out the applicable happy face, with choices of six faces ranging from very happy to very sad.

\section{Sleep-related breathing disorders}

Children with CP have a higher risk of sleep-related disorders than typically developing children [27]. Bensmail et al. showed that patients with severe spasticity due to spinal cord injury and multiple sclerosis, treated with ITB by bolus administration, showed an increased respiratory disturbance index (the number of apneas/ hypopneas per hour of sleep) $[28,29]$. Polysomnography is the gold standard when measuring sleep-related breathing disorders (SRBD). However, this time-consuming and burdensome diagnostic test is not practical for children participating in research [30]. A subscale of the Pediatric Sleep Questionnaire was developed to measure SRBD. This scale consists of 22 items and can be completed in five minutes. Sensitivity and specificity are high $(81 \%$ and $87 \%)$. The scale is positive for a high risk of SRBD when there are 8 or more positive answers to the 22 question items $(\geq 33 \%)$ [30]. As the burden of a questionnaire is low for the patients and their families, as compared to polysomnography, we will use this questionnaire to determine if ITB changes the risk of SRBD. 


\section{Classification}

For classification of the severity of motor abilities we will use the GMFCS [31,32] and the Manual Ability Classification System (MACS) [33,34]. GMFCS and MACS classification will be scored at baseline, 3 months and at twelve months. In a study by Voorman and co-workers, $74 \%$ of children with CP had restrictions in communication [35]. Since many children with severe CP (GMFCS IV and V) cannot speak, assessment of language abilities cannot be based on speech production. In addition, language comprehension skills are difficult to assess in children with severe CP. Therefore, we will use the Computer-Based Instrument for Low motor Language Testing (C-BiLLT) to measure comprehension of spoken language at baseline. The validity of this instrument has been tested [36]. We do not expect changes in outcomes on the C-BiLLT with ITB treatment since comprehension of spoken language is highly correlated with cognition, and cognition is not effected by ITB treatment. We will use the outcome of the C-BiLLT as a patient characteristic.

Magnetic resonance imaging (MRI) will be used to classify the severity of damage to the gray matter structures (cortex and basal ganglia) and white matter (loss of white matter and gliosis). The severity of brain damage on MRI can be classified in three groups; mild, moderate and severe. The mild pattern includes involvement of nucleus lentiformis and ventro-lateral thalamus, the moderate pattern includes additional involvement of the peri-central region and the severe pattern includes additional involvement of the entire thalamus and hippocampus. In the mild and moderate damage groups, infants suffer from the dyskinetic form of cerebral palsy, whereas the severe type of damage frequently produces purely spastic paresis [3]. MRI studies of the brain are necessary to confirm the diagnosis of cerebral palsy. If MRI studies have been conducted previously, these studies will be critically assessed. If the quality is good, the MRI will be accepted and no further imaging is needed. If the quality of the MRI images is poor, the patient will undergo a new MRI including diffusion tensor imaging (DTI). If a patient had not yet undergone a MRI, one will be made including DTI. Adding DTI images to a regular MRI scan will require an extra scanning time of approximately four minutes. In this patient category MRI imaging has to be done under general anesthesia, since dystonic movements interfere with MRI quality.

\section{Other study parameters}

To assess the safety of ITB treatment, side effects and complications will be closely monitored. The complication rate will be calculated by dividing the number of complications by the duration in years of pump implantation.
Functional abilities at baseline will be assessed by the Paediatric Evaluation of Disability Inventory (PEDI) and will be repeated after three and twelve months. The PEDI was developed for children from six months to seven and a half years of age and assesses skills in mobility, self-care and social function. It can also be used for older children if their functional abilities are expected to be below the level of a child of seven and a half years old. The functional scales indicate if children with disability are able or unable to perform certain activities. Separate measures assess the degree of caregiving assistance and equipment modification that is needed to accomplish complex functional skills. Scores on the caregiver assistance scale are noted on a range from independent to maximal assistance and modification scores are scored as none, child-oriented, rehabilitation-oriented or extensive $[37,38]$. The PEDI will be administered through direct assessment by a therapist.

\section{Randomization, blinding and treatment allocation}

Subjects will be randomized in two groups by block randomization. Randomization will be done by the pharmacies of the VUMC and the MUMC. The pharmacist will be the only holder of the code for randomization. In case of emergency, the code will be accessible 24 hours per day and 7 days per week via either the pharmacist of the VUMC or MUMC or by opening a sealed envelope containing the subject's group, available at the departments concerned. Allocation will be concealed, and the researchers, assessors and the physician responsible for pump filling will be blinded.

\section{Premature termination}

\section{Withdrawal of individual subjects}

Subjects can end participation in the study at any time without providing a reason and without consequences for their future treatment in the clinic. The investigator can decide to withdraw a subject from the study for urgent medical reasons. Subjects withdrawn from the study will continue their regular follow-up outside of the study protocol. Subjects will be replaced if they withdraw before pump implantation has taken place. Subjects who withdraw after pump implantation has taken place will not be replaced. With subject agreement, a final assessment will take place before definite ending of participation. These subjects will not be included in the analysis but we will present a fact sheet including their information.

\section{Data safety monitoring board}

A data safety monitoring board will be formed and will meet periodically to review aggregate and individual subject data related to safety, data integrity and overall conduct of the trial. They will assess the risk/benefit 
balance, including a statistical analysis if necessary. Depending upon this assessment, the board will provide recommendations to continue, adapt or terminate the trial.

\section{Statistics \\ Descriptive statistics}

Patient characteristics will be described. Gender distribution will be compared between the ITB and placebo group using a Chi-square test. Mean age between the ITB and placebo group will be compared using an independent samples t-test. Means and standard deviation (SD) of the PEDI scores, C-BiLLT scores, GAS t-score, BAD score, SPAT score and mean EMG activity at baseline will be tabulated. Means and SD of the GAS t-score, BAD score, DIS score, SPAT score and mean EMG activity at follow up will be separately tabulated per group.

\section{Univariate analysis}

The GAS t-scores, BAD score, SPAT score and EMG activity in the placebo and ITB group will be compared using an independent samples t-test. If the assumption of normality appears not to be valid, the non-parametric Mann-Whitney test will instead be used. A p-value of 0.05 is considered statistically significant for this primary analysis. To assess within-group changes in means between follow-up times and baseline GAS t-scores, BAD score, DIS score, SPAT score and EMG activity, a paired t-test or non-parametric Wilcoxon test will be used (depending on whether the normality assumption is valid). In these analyses, a p-value of 0.05 is considered to be statistically significant.

\section{Multivariate analysis}

A multivariate analysis will be used to determine the effect of ITB treatment (primary: functional outcome; secondary: dystonia and the interaction with GMFCS, MACS, MRI classification and use of co-medication).

\section{Discussion}

We anticipate that the results of this study will allow evidence-based use of intrathecal baclofen in dystonic cerebral palsy.

\footnotetext{
Abbreviations

CP: Cerebral palsy; GMFCS: Gross motor functioning classification system; VUMC: VU University medical center; MACS: Manual ability classification System; EMG: Electromyography; MUMC: Maastricht University medical center; ITB: Intrathecal baclofen; MRI: Magnetic resonance imaging; SD: Standard deviation; PEDI: Pediatric evaluation of disability inventory; RM: Repetitive movements; GAS: Goal attainment scaling; BAD: Barry albright dystonia scale; DIS: Dyskinesia impairment scale; C-BiLLT: Computer based instrument for low motor language testing; dti: Diffusion tensor imaging; VAS: Visual analogue scale; ICF: International classification of functioning, disability and health
}

\section{Competing interests}

There are no competing interests or financial competing interests.

\section{Authors' contributions}

LB participated in the design and drafted the manuscript. JB and RV participated in the design and first drafts of the manuscript. JV, KB, DS, VG, WO, RS, EF, JG, PV, OT participated in reviewing the design. All authors participated in the reviewing process and approved the final manuscript.

\section{Acknowledgements}

We gratefully acknowledge the Phelps Stichting voor Spastici (nr 2011037), the Johanna KinderFonds and the Kinderrevalidatie Fonds de Adriaan Stichting (both nr 2011/0035) and the RevalidatieFonds (nr R2011032) for funding of this project.

\section{Author details}

'Department of Rehabilitation Medicine, VU University Medical Center, Postbus 7057, 1007, MB Amsterdam, The Netherlands. ${ }^{2}$ Department of Child Neurology, Maastricht University Medical Center, Amsterdam, The Netherlands. ${ }^{3}$ Department of Neurosurgery, VU University Medical Center, Amsterdam, The Netherlands. ${ }^{4}$ Department of Clinical Neurophysiology, VU University Medical Center, Amsterdam, The Netherlands. ${ }^{5}$ Department of Neurology, VU University Medical Center, Amsterdam, The Netherlands. ${ }^{6}$ Department of Epidemiology and Biostatistics, VU University Medical Center, Amsterdam, The Netherlands. 'Department of Neurosurgery, Maastricht University Medical Center, Amsterdam, The Netherlands. ${ }^{8}$ Department of Child Neurology, Neuroscience Campus Amsterdam, VU University Medical Center, Amsterdam, The Netherlands.

Received: 23 September 2013 Accepted: 3 October 2013

Published: 28 October 2013

\section{References}

1. Hagberg B, Hagberg G, Olow I, von Wendt L: The changing panorama of cerebral palsy in Sweden. V. The birth year period 1979-82. Acta Paediatr Scand 1989, 78:283-290.

2. Bax M, Goldstein M, Rosenbaum PL, Levinton A, Paneth N, Dan B, Jacobsson B, Damiano D: Proposed definition and classification of cerebral palsy, April 2005. Dev Med Child Neurol 2005, 47:571-576.

3. Krägeloh-Mann I, Helber A, Mader I, Staudt M, Wolff M, Groenendaal F, DeVries L: Bilateral lesions of thalamus and basal ganglia: origin and outcome. Dev Med Child Neurol 2002, 44:477-484.

4. Jankovic J: Treatment of hyperkinetic movement disorders. The Lancet Neurology 2009, 8:844-856.

5. Sanger TD, Bastian A, Brunstrom J, Damiano D, Delgado M, Dure L, GaeblerSpira D, Hoon A, Mink JW, Sherman-Levine S, Wlty L: Child Motor Study Group. Prospective open-label clinical trial of trihexyphnidyl in children with secondary dystonia due to cerebral palsy. J Child Neurol 2007, 22:530-537.

6. Dan B, Motta F, Vles JS, Vloeberghs M, Becher JG, Eunson P, Gautheron V, Lutjen S, Mall V, Pascual-Pascual SI, Pauwels P, Roste GK: Consensus on the appropriate use of intrathecal baclofen (ITB) therapy in paediatric spasticity. Eur J Paediatr Neurol 2010, 14:19-28.

7. van Hulst BM, Tel PA, de Groot V, van Ouwerkerk WJ, Vermeulen RJ, Becher $J G$, Peerdeman SM: Complicaties bij kinderen met intrathecale baclofentherapie en (gerelateerde) verzorgertevredenheid. Tijdschrift voor kindergeneeskunde 2009, 77:191-197.

8. Ward A, Hayden S, Dexter M, Scheinberg A: Continuous intrathecal baclofen for children with spasticity and/or dystonia: Goal attainment and complications associated with treatment. J Paediatr Child Health 2009, 45:720-726

9. Albright AL, Barry MJ, Fasick P, Barron W, Shultz B: Continuous intrathecal baclofen infusion for symptomatic generalized dystonia. Neurosurgery 1996, 38:934-939.

10. Albright AL, Barry MJ, Painter MJ, Shultz B: Infusion of intrathecal baclofen for generalized dystonia in cerebral palsy. J Neurosurg 1998, 88:73-76.

11. Albright AL, Barry MJ, Shafron DH, Ferson SS: Intrathecal baclofen for generalized dystonia. Dev Med Child Neurol 2001, 43:652-657.

12. World Health Organisation: International Classification of Functioning. Geneva: Disability and Health; 2001. Ref Type: Catalog. 
13. Turner-Stokes L: Goal attainment scaling (GAS) in rehabilitation: a practical guide. Clin Rehabil 2009, 23:362-370.

14. Bovend'Eerdt TJH, Botell RE, Wade DT: Writing SMART rehabilitation goals and achieving goal attainment scaling: a practical guide. Clin Rehabil 2009, 23:352-361.

15. Steenbeek D, Ketelaar M, Galama K, Gorter JW: Goal attainment scaling in paediatric rehabilitation: a critical review of the literature. Dev Med Child Neurol 2007, 49:550-556.

16. Steenbeek D, Ketelaar M, Galama K, Gorter JW: Goal attainment scaling in paediatric rehabilitation: a report on the clinical training of an interdisciplinary team. Child: care, health and development 2008, 34:521-529.

17. Steenbeek D, Ketelaar M, Lindeman E, Galama K, Gorter JW: Interrater reliability of goal attainment scaling in rehabilitation of children with cerebral palsy. Arch Phys Med Rehabil 2010, 91:429-435.

18. Choate R, Smith A, Cardillo JE, Thompson L: Training in the use of Goal Attainment Scaling. Community Ment Health J 1981, 17:171-181.

19. Steenbeek D, Meester-Delver A, Becher JG, Lankhorst GJ: The effect of botulinum toxin type $A$ treatment of the lower extremity on the level of functional abilities in children with cerebral palsy: evaluation with goal attainment scaling. Clin Rehabil 2005, 19:274-282.

20. Monbaliu E, Ortibus E, De Cat J, Dan B, Heyrman L, Prinzie P, De Cock P, Feys H: The Dyskinesia Impairment Scale: a new instrument to measure dystonia and choreoathetosis in dyskinetic cerebral palsy. Dev Med Child Neurol 2012, 54:278-283.

21. Barry MJ, VanSwearingen JM, Albright AL: Reliability and responsiveness of the Barry-Albright Dystonia scale. Dev Med Child Neurol 1999, 41:404-411.

22. Hoving MA, van Kranen-Mastenbroek VHJM, van Raak EPM, Spincemaille GHJJ, Hardy ELM, Vles JSH: On behalf of the Dutch Study Group on Child Spasticity. Placebo controlled utility and feasibility study of the H-reflex and flexor reflex in spastic children treated with intrathecal baclofen. Clin Neurophysiol 2006, 117:1508-1517.

23. Bour LJ, Ongerboer de Visser BW, Koelman JHTM, van Bruggen GJ, Speelman JD: Soleus H-reflex tests in spasticity and dystonia: A computerized analysis. J Electromyogr Kinesiol 1991, 1:9-19.

24. Becher JG, Doorenbosch C, Folmer K, Scholtes VAB, Voorman JM, Wolterbeek N: Handleiding standaard lichamelijk onderzoek bij kinderen met een centraal motorische parese. Amsterdam: Reed business; 2011.

25. Scholtes VAB, Dallmeijer AJ, Becher JG: The spasticity test: a clinical instrument to measure spasticity in children with cerebral palsy. The effectiveness of multilevel botulinum toxin type $A$ and comprehensive rehabilitation in children with cerebral palsy [dissertation]. Amsterdam: VU University Medical Center; 2007.

26. Scholtes VAB, Becher JG, Beelen A, Lankhorst GJ: Clinical assessment of spasticity in children with cerebral palsy: a critical review of available instruments. Dev Med Child Neurol 2006, 48:64-73.

27. Sandella DE, O'Brien LM, Shank LK, Warschausky SA: Sleep and quality of life in children with cerebral palsy. Sleep Med 2011, 12:252-256.

28. Bensmail D, Quera Salva MA, Roche N, Benyahia S, Bohic M, Denys P, Bussel $B$, Lofaso F: Effect of intrathecal baclofen on sleep and respiratory function in patients with spasticity. Neurology 2006, 67:1432-1436.

29. Bensmail D, Marquer A, Roche N, Godard A, Lofaso F, Quera Salva MA: Pilot study assessing the impact of intrathecal baclofen administration mode on sleep-related respiratory parameters. Arch Phys Med Rehabil 2012, 93:96-99.

30. Chervin RD, Hedger K, Dillon Jl, Pituch KJ: Pediatric Sleep Questionnaire (PSQ): validity and reliability of scales for sleep-disordered breathing, snoring, sleepiness and behavioral problems. Sleep Med 2000, 1:21-32.

31. Palisano RJ, Hanna SE, Rosenbaum PL, Russel DJ, Walter EP, Raina PS, Galuppi BE: Validation of a model of gross motor function for children with cerebral palsy. Phys Ther 2000, 80:974-985.

32. Wood E, Rosenbaum PL: The gross motor function classification system for cerebral palsy: a study of reliability and stability over time. Dev Med Child Neurol 2000, 42:292-296.

33. Eliasson AC, Krumlinde-Sundholm L, Rösblad B, Beckung E, Arner M, Ohrvall AM, Rosenbaum PL: The Manual Ability Classification System (MACS) for children with cerebral palsy: scale development and evidence of validity and reliability. Dev Med Child Neurol 2006, 48:549-554.

34. Morris C, Kurinczuk JJ, Fitzpatrick R, Rosenbaum PL: Reliability of the manual ability classification system for children with cerebral palsy. Dev Med Child Neurol 2006, 48:950-953.
35. Voorman JM, Dallmeijer AJ, van Eck M, Schuengel C, Becher JG: Social functioning and communication in children with cerebral palsy: association with disease characteristics and personal and environmental factors. Dev Med Child Neurol 2009, 52:441-447.

36. Geytenbeek JJM, Heim MMJ, Vermeulen RJ, Oostrom KJ: Assessing comprehension of spoken language in nonspeaking children with cerebral palsy: application of a newly developed computer based instrument. Augment Altern Commun 2010, 26:97-107.

37. Feldman AB, Haley SM, Coryell J: Concurrent and construct validity of the Pediatric Evaluation of Disability Inventory. Phys Ther 1990, 70:602-610.

38. Haley SM, Coster WJ, Ludlow LH, Haltiwanger JT, Andrellos PJ: Pediatric Evaluation of Disability Inventory (PEDI): development, standardization and administration manual. Boston, MA: PEDI Research Group, Department of Rehabilitation Medicine; 1992

doi:10.1186/1471-2431-13-175

Cite this article as: Bonouvrié et al: Intrathecal baclofen treatment in dystonic cerebral palsy: a randomized clinical trial: the IDYS trial. BMC Pediatrics 2013 13:175.

\section{Submit your next manuscript to BioMed Central and take full advantage of:}

- Convenient online submission

- Thorough peer review

- No space constraints or color figure charges

- Immediate publication on acceptance

- Inclusion in PubMed, CAS, Scopus and Google Scholar

- Research which is freely available for redistribution 